\title{
Antioxidant potential study of some synthesized $N$-heterocycles
}

\author{
Mohammad Mamun Hossain, Sukanta Kumar Shaha and Foysal Aziz \\ Department of Chemistry, Jahangirnagar University, Savar, Dhaka 1342, Bangladesh. \\ e-mail: chemmamun2@yahoo.com
}

\begin{abstract}
Nitrogen containing heterocyclic compounds such as oxindoles especially isatins and their derivatives have excellent biological properties such as anti-inflammatory, antimicrobial, anticancer activity. In addition, this is first time ever we would like to report the antioxidant properties of the said class of compounds. To determine such an important bioactivity a number of halogenated oxindoles 1-3, their diaza-biphenyl derivatives (4-6), ring extended biphenyl (7) and bis-amide (8) were synthesized by classical heating method. The antioxidant activity of all the synthesized compounds was screened by DPPH method with respect to ascorbic acid. In our present investigation some of the synthesized compounds $(\mathbf{1}, \mathbf{2}, \mathbf{3}, \mathbf{5}$ and $\mathbf{6})$ were found to be active.
\end{abstract}

\section{Introduction}

Oxindoles particularly isatins and their derivatives are important class of compounds due to their excellent biological effects, including antifungal, antiviral, and antiproliferative activities ${ }^{1}$. In addition, these compounds are very useful synthetic intermediates and can function as suitable building blocks to synthesize some other biologically active compounds such as natural products. Hence a significant rising research interest in the design of oxindoles as drugs is currently observed in the field of medicinal organic chemistry. The Schiff bases and spiro-thiadiazoline derivatives of isatins have shown remarkable biological activities, which is already reported ${ }^{2-4}$. On the other hand, the consumption of citrus fruits, vegetables, red wines, juices, etc. provides protection against diseases, including cancer, cardiovascular, cerebrovascular and many other diseases ${ }^{5,6}$. A variety of natural substances such as flavonoids from many other plants are used by humans as antioxidants to trap and deactivate damaging oxygen-centred radicals. This protection can be explained by the capacity of these antioxidants to scavenge free radicals, which are responsible for the oxidative damage of lipids, proteins and nucleic acids ${ }^{7-9}$. It is wroth to be noted here that antioxidant-enriched sources contain mainly vitamin $\mathrm{C}$, which is chemically a cyclic ester (lactone). Since oxindoles have very close structural similarity, cyclic amide (lactam) with antioxidant compounds such as vitamin $\mathrm{C}$, it would not be surprising if oxindoles show antioxidant properties. Therefore, it grew our interest to synthesize some indole-based diaza-biphenyl compounds for the test to show any antioxidant activity. Accordingly numerous diaza-biphenyl compounds were synthesized from the reaction of oxindoles and ethylene/propylene diamine in fairly good yields. This is for the first time we report here the antioxidant properties of the synthesized compounds.

\section{Materials and Methods}

Synopsis of the synthetic route is shown in Scheme 1. The chemical structures of synthesized compounds are shown in Scheme 2.

Antioxidant potential: It is well-known that free radicals cause autoxidation of unsaturated lipids in food. In addition, antioxidants are known to interrupt the free-radical chain of oxidation and to donate hydrogen from phenolic hydroxy groups, thereby, forming stable free radicals, which do not initiate or propagate further oxidation of lipids ${ }^{10}$. The antioxidant potential of any compound can be determined on the basis of its scavenging activity of the stable 1,1-diphenyl-2-picryl hydrazyl (DPPH) free radical as described by Sadhu et al ${ }^{9}$. DPPH is a stable free radical containing an odd electron in its structure and usually utilized for detection of the radical scavenging activity in chemical analysis. The aliquot of the different concentrations $(5-500 \mu \mathrm{g} / \mathrm{mL})$ of the test sample is added to $3 \mathrm{~mL}$ of a $0.004 \%$ ethanolic solution of 


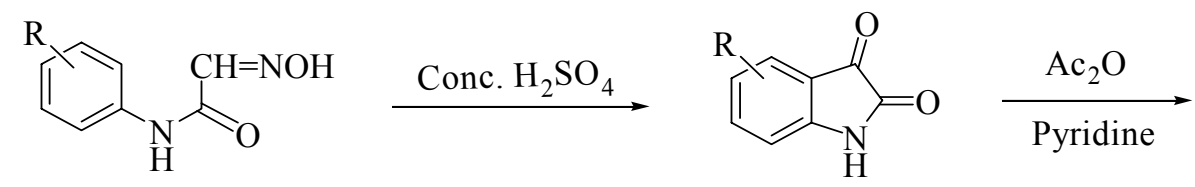

$\mathrm{R}=\mathrm{H}, \mathrm{Cl}, \mathrm{Br}$
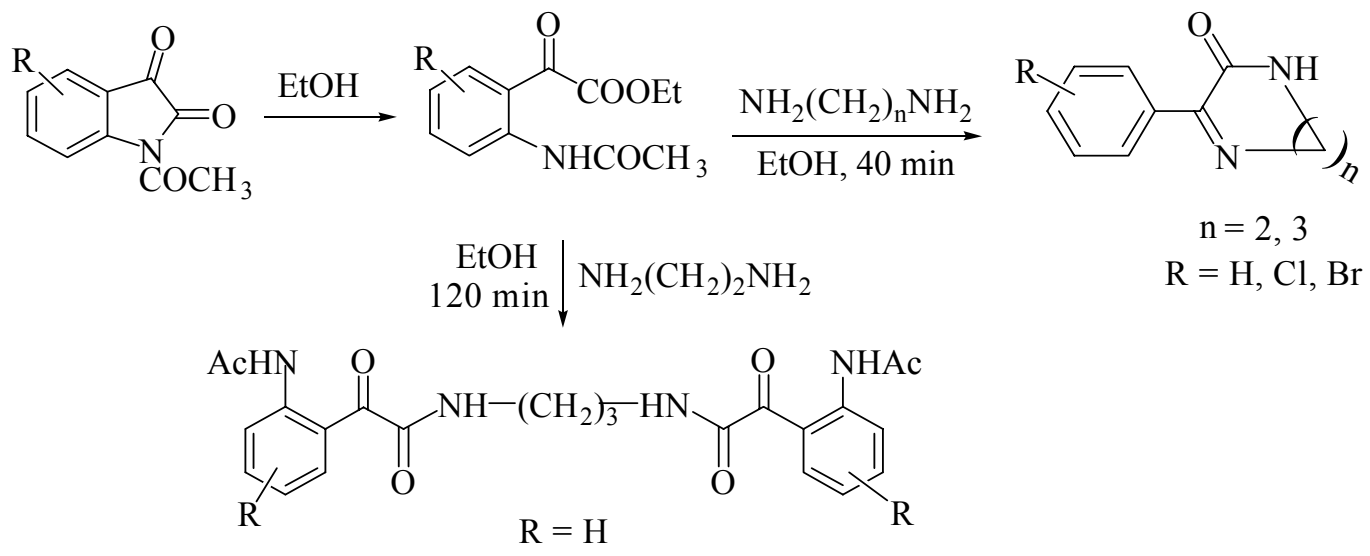

Scheme 1

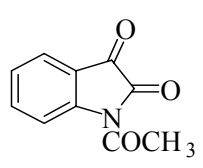

1<smiles>CC(=O)N1C(=O)C(=O)c2cc(Cl)ccc21</smiles>

2<smiles>COC(=O)c1ccc(Br)cc1C(=O)O</smiles>

3<smiles>COC(=O)Nc1ccccc1C1=C(C)CCNC1=O</smiles>

4<smiles>CC(=O)Nc1cc(Cl)ccc1C1=NCCNC1=O</smiles><smiles>C=NC1=C(c2ccc(Br)cc2NC(=O)OC)C(=O)NCC1</smiles>

6

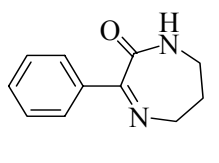<smiles>CNc1ccccc1C(=O)C(=O)NCCCCCNC(=O)C(=O)c1ccccc1NC</smiles>

\section{Scheme 2}

DPPH. Absorbance at $517 \mathrm{~nm}$ is determined after $30 \mathrm{~min}$, and $\mathrm{IC}_{50}$ (Inhibitory concentration $50 \%$ ) is also determined. $\mathrm{IC}_{50}$ value denotes the concentration of sample required to scavenge $50 \%$ of the DPPH free radicals (Scheme 3). The formula used for \%inhibition is as follows:

$\%$ inhibition $=($ Blank OD - Sample OD/Blank OD $) \times 100$<smiles>O=[N+]([O-])c1cc([N+](=O)[O-])c(NN(c2ccccc2)c2ccccc2)c([N+](=O)[O-])c1</smiles>

DPPH radical<smiles>O=C1OC(C(O)CO)C(O)=C1O</smiles>

Ascorbic Acid

\section{Scheme 3}

Sampling, screening and $I C_{50}$ calculation: At first 5 test tubes were taken to make aliquots of 5 different concentrations level $(5,10,50,100$ and 500 $\mu \mathrm{g} / \mathrm{mL}$ ). Tested sample and ascorbic acid were weighed 3 times and dissolved in ethanol to make the required concentrations by dilution technique. DPPH was weighed and dissolved in ethanol to make $0.004 \% \quad(w / v)$ solution. To make homogeneous solutions of the tested samples, magnetic stirrer was used. After making the desired concentrations, $2 \mathrm{~mL}$ of $0.004 \%$ DPPH solution was applied on each test tube by using pipette. The room temperature was recorded and kept the test tubes for 30 minutes in light exposure to complete the reactions. DPPH was also applied on the blank test tubes at the same time where only ethanol was taken as blank. After 30 min, absorbance of each test tube was determined by UV spectrophotometer. Then \%inhibitions were plotted against log concentration of each of the test sample. Then $\mathrm{IC}_{50}$ was calculated from the graph. The experiment was performed in duplicate and average absorption was noted for each concentration. Ascorbic acid was used as a positive control. Calculated antioxidant data of all the test samples were summarized in Table I. 
Table I: Antioxidant potential of the synthesized compounds

\begin{tabular}{ccl}
\hline Compound No. & $\mathrm{IC}_{50}$ Value & Remarks \\
\hline $\mathbf{1}$ & 673.61 & Moderately active \\
$\mathbf{2}$ & 398.27 & Highly active \\
$\mathbf{3}$ & 435.40 & Highly active \\
$\mathbf{4}$ & 4156.80 & Less active \\
$\mathbf{5}$ & 253.57 & Highly active \\
$\mathbf{6}$ & 1355.02 & Moderately active \\
$\mathbf{7}$ & 4323.33 & Less active \\
$\mathbf{8}$ & 3221.74 & Less active \\
\hline
\end{tabular}

Free radical scavenging mechanism of antioxidants: The 1,1-diphenyl-2-picrylhydrazyl radical has been widely used to evaluate the free radical scavenging capacity of different antioxidants $^{11-13}$. With this method it is possible to determine the antiradical power of an antioxidant activity by measurement of the decrease in the absorbance of DPPH at $517 \mathrm{~nm}$. Resulting from a color change from purple to yellow the absorbance decreased when the DPPH is scavenged by an antioxidant, through donation of hydrogen to form a stable DPPH molecule. In the radical form this molecule had an absorbance at $517 \mathrm{~nm}$, which disappeared after acceptance of an electron or hydrogen radical from an antioxidant compound to become a stable diamagnetic spin paired molecule ${ }^{14}$. The odd electron in the DPPH free radical gives a strong absorption maximum at 517 $\mathrm{nm}$ and is purple in color. The color turns from purple to yellow as the molar absorptivity (optical density) of the DPPH radical at $517 \mathrm{~nm}$ reduces from 9660 to 1640 when the odd electron of DPPH radical becomes paired with hydrogen radical from a free radical scavenging antioxidant to form the reduced DPPH-H. The resulting decolorization is stoichiometric with respect to the number of electrons captured.

\section{Results}

Antioxidant results of the synthesized compounds (1-8) with respect to ascorbic acid were reported in Table I. As per chemical structural features there were three different types of compounds synthesized under the study area. It is obvious that structural variation brings about the bioactivity and, of course, structural modification of molecules alters the biological activity in a regular trend. Without exception this has been reflected in the case of the acetylation of lactam resulted compounds 1-3, which showed upward tendency of antioxidant activity. Furthermore, substitution in the aromatic ring system with halogens like chlorine or bromine sharply enhanced the antioxidant potency. This is why compounds $\mathbf{2}$ and
3 were found to be more active than the compound 1. On the other hand, diaza-biphenyls (4-6) showed irregular trend in antioxidant activity. Compound $\mathbf{5}$ and 6 were attuned to show antioxidant activity because both of them were halogen substituted as well as comprised with similar structural features. But compound 5 was found to be the most efficacious antioxidant among all the listed compounds. Actually the antioxidant efficacy of any compound depends strongly on its reducing property and compound $\mathbf{5}$ might have the higher reducing potential.

\section{Discussion}

The present investigation emphasized mainly on two important things. Of these one is to the synthesis of molecules having $N$-heterocycles and the other is to determine their antioxidant efficacy. Nitrogen containing heterocyclic organic compounds having extra keto group show interesting chemical property as well as biological activity. ${ }^{15}$ The synthetic route of the compounds (18) was mainly based on the substitution, ring cleavage and consequent ring extension reactions through cyclization. Hence the synthetic pathway facilitated to introduce further $N$-atom in the ring system to provide diaza-biphenyls with $\mathrm{C}=\mathrm{O}$ moieties. Furthermore, each of the compounds contains a high percentage of nitrogen. Therefore, it might have enhanced the power to absorb free radicals, especially reactive oxygen and reactive nitrogen species (ROS and RNS). This finding is also concomitant with the earlier work. ${ }^{16}$ The screening of the synthesized compounds through Structure Activity Relationship (SAR) showed that different indoxyls such as isatins were not so pronounce in their activity. But the $N$-acetyl derivatives $(\mathbf{2}, \mathbf{3})$ obtained from isatins by means of acetylation had shown greater propensity in showing antioxidant potentiality. This is presumably due to the cleavage of $\mathrm{CH}_{3} \mathrm{CO}-\mathrm{N}$ bond in a hemolytic fashion is rather easier than $\mathrm{N}-\mathrm{H}$ bond cleavage because of the greater stability of the corresponding $\mathrm{CH}_{3} \mathrm{CO}$ radical. On the other hand the cleavage of the lactam moieties and the consequent cyclization provided with diazabiphenyls (4-6), which were found to be anomalous in showing antioxidant activity. It is very clear from our present finding that heterocyclic systems with halogen substituted pattern (Cl- or Br-) show greater antioxidant property. In every case it was observed that molecules with halogen (bromine/chlorine) substitution such as compound 2, 3, 5 and $\mathbf{6}$ were found to be more active than their un-substituted counterpart. Compound $\mathbf{5}$ and $\mathbf{6}$ were compatible to show antioxidant activity 
because both of them were halogen substituted as well as comprised with similar structural features. Both compounds have N-H bonding in the diaza ring system so that hydrogen radical could be generated and the ring-nitrogen radical can scavenge the damaging radicals to prevent further oxidation. But compound 5 was found to be the most efficacious antioxidant among all compounds. Although we don't have the exact clarification with regard to its greater activity, we have some logical arguments in favor of the fact. Actually diazabiphenyl ring is responsible to initiate the free radical scavenging activity by means of reactive nitrogen species (RNS) as mentioned before. It is thought that the chlorine atom because of its lone pair electron as well as its electronegativity power enhanced the formation and subsequent stabilization of the nitrogen-ring radical through intervening aromatic system in case of compound 5. Due to extra stabilization, radical obtained from compound 5, would have higher aptitude to trap free radical in a faster rate than the other similar type of molecules.

\section{References}

1. Giselle C, Ferreira A. Oxindoles and copper complexes with oxindole derivatives as potential pharmacological agents. J Braz Chem Soc. 2006; 17: 1-22.

2. Hossain MM, Islam N, Khan R, Islam MR. Cytotoxicity study of dimethylisatin and its heterocyclic derivatives. Bangladesh J Pharmacol. 2007; 2: 66-70.

3. Islam MR, Abedin J, Khayer K. Synthesis of some 5spiro-(5'-methylisatin)-4- $N$-acetyl-2-acetylamino- $\Delta^{2}$ 1,3,4-thiadiazoline and 5-spiro-(5'-methylisatin)-4-Nacetyl-2-(5-methylisatin-3-hydrazino)- $\Delta^{2}-1,3,4$ thiadiazolines. Indian J Chem. 2001; 40B: 240-247.

4. Islam MR, Abedin J, Hossain MM, Duddeck H. Synthesis of 1-methyl bis-dioxopyrrolino$\left[2^{\prime}, 3\right.$ ' $: 2,3 ; 2$ ', 3 '’:6,5] benzene and its heterocycles via thiocarbohydrazone, thiosemicarbazone. J Bangladesh Chem Soc. 1998; 11: 71-78.
5. Ames BM. Dietary carcinogens and anticarcinogens, oxygen radicals and degenerative diseases. Science 1983; 221: 1256-63

6. Steinberg D. Antioxidants and atherosclerosis: A current assessment. Circulation 1991; 84: 1420-25.

7. Aruoma OI. Free radicals, oxidative stress, and antioxidants in human health and disease. J Am Oil Chem Soc. 1998; 75: 199-212.

8. Wang H, Cao G, Prior RL. Total antioxidant capacity of fruits. J Agric Food Chem. 1996; 44: 701-05.

9. Sadhu SK, Okuyama E, Fujimoto H, Ishibashi M. Separation of Leucas aspera, a medicinal plant of Bangladesh, guided by prostaglandin inhibitory and antioxidant activities. Chem Pharm Bull.2003; 51: 59598.

10. Sherwin ER. Oxidation and antioxidants in fat and oil processing. J Am Oil Chem Soci. 1978; 55: 809-41.

11. Brand-Willams W, Cuvelier ME, Berset C. Use of a free radical method to evaluate antioxidant activity. Lebensmittel-Wissenschaft and Technologie. 1995; 28 : 25-30.

12. Espin JC, Soler-Rivas C, Wichers HJ. Characterization of total free radical scavenger capacity of vegetable oils and oil fraction using 2,2- diphenyl-1picrylhydrazyl radical. J Agric Food Chem. 2000; 48: 648-656.

13. Yu L. Free radical scavenging properties of conjugated linoleic acids. J Agric Food Chem. 2001; 49: 3452-56.

14. Mattha"us B. Antioxidant activity of extracts obtained from residues of different oilseeds. J Agric Food Chem. 2002; 50: 3444-52.

15. Islam MR, Mohsin M. Synthesis of isatin, 5-chloroisatin and their $\Delta^{2}$-1,3,4-oxadiazoline derivatives for comparative cytotoxicity study on brine shrimp. Bangladesh J Pharmacol. 2007; 2: 7-12.

16. Gomes A, Fernandes E, Garcia MB, Silva AM, Pinto DC, Santo CM, Cavaleiro JA, Lima JL. Cyclic voltametric analysis of 2-styrylchromones: relationship with the antioxidant activity. Bioorg Med Chem.2008; 16: 7939-43. 
